\title{
Entropy in Spacetime and Topological Hair
}

\author{
Young-Hwan Hyun ${ }^{1, \star}$ and Yoonbai Kim ${ }^{1, \star \star}$ \\ ${ }^{1}$ Department of Physics and Institute of Basic Science, Sungkyunkwan University, Suwon 16419, Republic \\ of Korea
}

\begin{abstract}
Global topological soliton of the hedgehog ansatz is added to de Sitter spacetime in arbitrary dimensions larger than three, and then thermodynamic law is checked at the cosmological horizon. All geometric and thermodynamic quantities are varied in the presence of a long-range interacting matter distribution with negative pressure, however the entropy-area relation is satisfied in the exact form. Its geometry involves deficit solid angle but maintains a single horizon which allows unique temperature normalization, different from the case of Schwarzschild-de Sitter spacetime.
\end{abstract}

\section{Introduction}

Thermodynamic laws are established as empirical laws through numerous tests. Since the entropyarea relation was proposed as a thermodynamic law in curved spacetime with a black hole horizon [1], it has contributed for establishing the black hole thermodynamics [2, 3], and has been an active area of research towards quantum gravity, particularly for counting the degrees of freedom and information loss. In the middle of the progress of the black hole thermodynamics, the entropy-area relation has been checked from representative idealized systems to realistic dirty situations. The relation was examined in various quantum gravity theories and also in many different systems of mass, spin, charges, or the number of dimensions [4]. Except for some higher-derivative gravity theories, the entropy-area relation remains to be fulfilled. This universality occurs even for black solutions with different horizon topologies and for the cosmological horizon [5]. Although the various methods including string theory repeatedly confirm the entropy-area relation, the universality never have proper explanation in the context of quantum gravity despite many empirical evidences [4].

In this work we add one more intriguing empirical evidence to investigate the problem of universality. As an attractive candidate, we propose non-BPS topological solitons (as additional nontrivial matter distribution) in the de Sitter spacetime (dS) to test the entropy-area relation. Nonlinear interactions are included to form topological solitons in arbitrary spatial dimensions and non-BPS nature implies complicated mutual interaction between multiple solitons. Despite of this complexity, since its matter distribution is controllable and possesses the topological stability, it is tractable to compute the entropy-area relation in the exact form.

This article is organized as follows. In Section 2, we consider a matter distribution of divergent energy $E \sim R^{d-3}$ and test the entropy-area relation on the cosmological horizon of dS with deficit

\footnotetext{
$\star$ e-mail: yhhyun@skku.edu

$\star \star$ e-mail: yoonbai@skku.edu
} 
solid angle. In Section 3, we consider a scalar field theory of $\mathrm{O}(d-1)$ symmetry and identify the source of this divergent energy as the global topological soliton of a hedgehog ansatz. We conclude in Section 4 with a few discussions.

\section{Entropy-area relation with deficit solid angle}

Suppose that there is a matter distribution of which energy-momentum tensor is

$$
-T^{t}{ }_{t}=-T_{r}^{r}=\cdots \sim \frac{d-2}{2} \frac{v^{2}}{r^{2}},
$$

where the coefficient is chosen for later convenience. For $d \geq 3$, the energy in flat spacetime is divergent as the spatial volume goes to infinity,

$$
E \sim \int^{R} d r r^{d-2}\left(-T_{t}^{t}\right) \sim \int^{R} d r r^{d-2} \frac{1}{r^{2}} \Rightarrow\left\{\begin{array}{c}
\stackrel{d=3}{\sim} \ln R \\
\underset{d>3}{\sim} R^{d-3}
\end{array}\right\} \stackrel{R \rightarrow \infty}{\rightarrow} \infty .
$$

This $R^{d-3}$ divergence for $d>3$ seems not serious in the $d$-dimensional de Sitter spacetime $\left(\mathrm{dS}_{d}\right)$ in which the vacuum energy is growing with larger power, $E_{\Lambda} \sim R^{d-1}$. In fact, the $1 / r^{2}$-term is that of the natural largest power among various terms with different powers lower than the zeroth power (constancy) of the vacuum energy density, and thus seems naturally allowable as a matter distribution in $\mathrm{dS}_{d}$. A question at the moment is to find a possible field theoretic candidate which can be identified as a natural source giving such energy distribution (1) but will be discussed in the next section.

Introduce a metric in $\mathrm{dS}_{d}(d>3)$

$$
d s^{2}=-e^{2 \Omega(r)} A(r) d t^{2}+\frac{d r^{2}}{A(r)}+r^{2} d \Omega_{d-2}^{2},
$$

and then the solution of Einstein equation coupled to the aforementioned energy-momentum tensor in (1) is

$$
\Omega(r) \stackrel{\text { set }}{=} 0, \quad A(r)=-\left(\frac{r}{\ell}\right)^{2}+1-\delta-\frac{C}{r^{d-3}}
$$

where $\delta=\frac{8 \pi G v^{2}}{d-3}$. Since our main goal is to test the entropy-area relation in the presence of the matter of which distribution is relatively simple, we set $C=0$ to deal without creating a new black hole horizon. Performing a coordinate transformation, $d t^{\prime}=\sqrt{1-\delta} d t, \quad d r^{\prime}=\frac{d r}{\sqrt{1-\delta}}, \quad d \Omega_{d-2}^{\prime}=$ $(1-\delta) d \Omega_{d-2}$, the metric returns to that of pure $\mathrm{dS}_{d}$ with the different range of solid angle variables, which leads to the deficit solid angle.

To address the question whether or not the entropy-area relation is sustained for non-vanishing $\delta$, we begin by reading the location of the horizon $r_{\mathrm{h}}=\sqrt{1-\delta} \ell$ and thus the area of the horizon is decreased by the factor $(1-\delta)^{\frac{d-2}{2}}$,

$$
\mathcal{A}_{\delta \mathrm{dS}}^{\mathrm{h}}=\ell^{d-2} \Omega_{d-2}(1-\delta)^{\frac{d-2}{2}}=\mathcal{A}_{\mathrm{dS}}^{\mathrm{h}}(1-\delta)^{\frac{d-2}{2}},
$$

where $\Omega_{d-2}$ is the volume of a unit $(d-2)$-sphere. The surface gravity at the horizon $\kappa\left(r_{\mathrm{h}}\right)=|d A / d r|_{r_{\mathrm{h}}} / 2$ fixes the temperature under the standard normalization [6, 7]. The corresponding thermodynamic law with temperature and pressure accompanies the change of volume [6]

$$
T_{\delta \mathrm{dS}} \mathrm{d} S_{\delta \mathrm{dS}}=\mathrm{d}\left(-E_{\delta \mathrm{dS}}\right)+P_{\delta \mathrm{dS}} \mathrm{d}\left(-V_{\delta \mathrm{dS}}\right)
$$


where $-E_{\delta \mathrm{dS}}$ counts the negative energy and the surplus angle behind the horizon [5]. Note that there is a minus sign in front of the volume as well, since it accounts for the change of the volume of the inaccessible region [6]. According to the thermodynamic law (6), straightforward computation of the energy and the work inside the horizon results in the following entropy difference,

$$
\mathrm{d} S_{\delta \mathrm{dS}}=\frac{A_{\mathrm{dS}}}{4 G}\left(-\frac{d-2}{2}\right)(1-\delta)^{\frac{d-4}{2}} \mathrm{~d} \delta
$$

Though every thermodynamic quantity is changed by the matter distribution and its signal appears through deficit solid angle, the entropy-area relation is exactly satisfied irrespective of the change,

$$
S_{\delta \mathrm{dS}}=S_{\mathrm{dS}}+\Delta S_{\delta \mathrm{dS}}=S_{\mathrm{dS}}+\int_{S(\delta=0)}^{S(\delta)} d S_{\delta \mathrm{dS}}=\frac{\mathcal{A}_{\mathrm{dS}}^{\mathrm{h}}}{4 G}(1-\delta)^{\frac{d-2}{2}}=\frac{\mathcal{A}_{\delta \mathrm{dS}}^{\mathrm{h}}}{4 G} .
$$

In this section a matter distribution of long-ranged energy density and pressure is added to $\mathrm{dS}_{d}$. Then the corresponding geometry includes deficit solid angle but does not create a new black hole horizon in addition to the cosmological horizon. Though all thermodynamic quantities, e.g., area, temperature, energy, pressure, and entropy, are changed in $\delta \mathrm{dS}_{d}$ from those in pure $\mathrm{dS}_{d}$, we show that the exact entropy-area relation holds irrespective of the value of the deficit solid angle. This test supports the claimed universality of the entropy-area relation in the presence of interacting non-BPS matter.

\section{Global topological soliton of hedgehog ansatz as a source}

Once we look at the distribution of energy-momentum tensor in (1), it seems difficult not to ask a question on a possible candidate of such distribution. In this section let us identify a natural source of the matter of which energy-momentum tensor is given by (1). To find the origin in the framework of field theory, the action of the $\mathrm{O}(d-1)$ scalar fields $\phi^{i}(i, j, \cdots=1,2, \ldots, d-1)$ is considered

$$
S_{\phi}=\int d^{d} x \sqrt{-g}\left[-\frac{g^{\mu \nu}}{2} \partial_{\mu} \phi^{i} \partial_{\nu} \phi^{i}-\frac{\lambda}{4}\left(\phi^{2}-v^{2}\right)^{2}\right],
$$

where $\phi^{i} \equiv \hat{\phi}^{i} \phi$ with $\hat{\phi}^{i} \hat{\phi}^{i}=1$ and a quartic scalar potential $V(\phi)$ with spontaneous symmetry breaking is chosen for simplicity. The configuration under the hedgehog ansatz, $\phi^{i}=\hat{r}^{i} \phi(r)$, supports a topologically stable soliton and its topological charge is given by the winding between the $S^{d-2}$ of broken vacua and the spatial $\mathrm{S}^{d-2}$ at the horizon $r_{\mathrm{h}}$.

To solve the scalar equation and the non-vanishing components of the Einstein equation, we apply the following boundary conditions.

$$
\phi(0)=0, \quad \phi\left(r_{\mathrm{h}}\right)=\phi_{\mathrm{h}}(<v) \stackrel{\ell \gg 1 / \sqrt{\lambda} v}{\longrightarrow} v, \quad A(0)=1, \quad \Omega\left(r_{\mathrm{h}}\right)=0 .
$$

The first condition is for a nonsingular field configuration under the hedgehog ansatz, and the second condition supports the topological soliton. The presumed dS horizon $r_{\mathrm{h}}$ seems subtle because of possible existence of the short scalar hair. When the size of the horizon $\left(r_{\mathrm{h}} \sim \ell\right)$ is much larger than that of the core of a topological defect $\left(r_{\text {core }} \sim 1 / \sqrt{\lambda} v\right)$, the scalar amplitude takes the value of the vacuum. Since no additional mass was assumed, that is $C=0$ in (4), $A(r)$ becomes the metric of flat spacetime at the origin. The last condition is for the correct normalization of temperature at the $\mathrm{dS}$ horizon, so the condition is assigned for later convenience without losing generality. 
Approximate solution near but inside the dS horizon is attempted under the assumption that $r$ is much larger than the core radius $1 / \sqrt{\lambda} v$,

$$
\begin{aligned}
& \phi(r) \approx v-\frac{d-2}{2\left[1+\frac{3-d}{(\sqrt{\lambda} v \ell)^{2}}\right]} \frac{v}{(\sqrt{\lambda} v r)^{2}}+\cdots, \\
& A(r) \approx-\left(\frac{r}{\ell}\right)^{2}+1-\delta+\cdots, \\
& \Omega(r) \approx-\frac{(d-2)(d-3)}{4\left[1+\frac{3-d}{(\sqrt{\lambda} v \ell)^{2}}\right]^{2}} \delta \frac{1}{(\sqrt{\lambda} v r)^{4}}+\cdots .
\end{aligned}
$$

Since the expansion assumed the scale $\ell \gg 1 / \sqrt{\lambda} v$, the formula $1+(3-d) / \lambda v^{2} \ell^{2}$ in (11) and (13) is always positive so that $\phi(r)$ and $\Omega(r)$ become monotonically increasing at interior region of the horizon. Then the non-vanishing component of the Einstein equation leads to the energy-momentum tensor in (1) exactly irrespective of the spacetime dimensions $d$. Since the deficit solid angle must be smaller than the solid angle itself or equivalently $\delta<1$, the scale of the vacuum expectation value $v$ is constrained by the $d$-dimensional Planck scale, $v<\sqrt{\frac{d-3}{8 \pi G}}$. The radial component of pressure also has a long tail,

$$
T_{r}^{r}=-\frac{d-2}{2} \frac{\phi^{2}}{r^{2}}+\frac{A}{2}\left(\frac{d \phi}{d r}\right)^{2}-V \underset{r \sim r_{\mathrm{h}}}{\stackrel{\phi \approx v}{\rightarrow}}-\frac{d-2}{2} \frac{v^{2}}{r^{2}},
$$

which approaches (1) near the cosmological horizon. Negativity of the pressure explains the decrease of the area of the cosmological horizon (5) by adding this global topological soliton.

Intriguingly enough, the origin of divergent energy is identified by the global topological soliton of the hedgehog ansatz. In $d=4$ dimensions, the obtained topologically stable global soliton is the global monopole with linearly divergent energy [8]. The signature of energy density leads to a repulsive gravitational potential [9], which explains impossibility of formation of a new horizon in addition to the cosmological horizon. Though the energy of a global topological soliton diverges as $E \sim R^{d-3}$, it can easily be produced through a period of cosmological phase transition by consuming a part of vacuum energy [10].

\section{Conclusion}

Global topological soliton of the hedgehog ansatz is considered in $\mathrm{O}(d-1)$ symmetric scalar field theory in the background of $\mathrm{dS}_{d}$. The entropy-area relation is confirmed in the presence of divergent energy of the global solitonic object through an easier analysis without creating an additional horizon. This result adds a circumstantial evidence on the issue of universality of the entropy-area relation [4]. Since the matter distribution of the global topological solitons is stable and makes the problem tractable, many of them can provide good candidates to test and establish thermodynamic relations in curved spacetime.

We conclude with a few brief discussions:

1. Though our analysis was performed without mass term, $C=0$ in (4), in order not to create an additional horizon, it is also intriguing to examine the entropy-area relation with both global topological soliton and usual matter of $C \neq 0$, the analysis of the entropy-area relation in $\delta \mathrm{SdS}_{d}$ including the Nariai limit. 
2. Three-dimensional case was excluded because the $1 / r^{2}$ term in $\mathrm{dS}_{3}$ is nothing but the energy density of the electrostatic field of point charge, $-T^{t}{ }_{t} \sim \mathbf{E}^{2} \sim 1 / r^{2}$, which leads to a logarithmic term $A(r) \propto \ln r$ as in (2). Different from $d>3$, the deficit angle $2 \pi \delta$ is obtained by shifting the constant term in the metric, of which physical origin is a localized point source [11]. The entropy-area relation in $\delta \mathrm{dS}_{3}$ by utilizing the deficit angle was checked in Ref. [12].

3. In this work, since the spacetime symmetry is broken in the radial direction and includes non-trivial red-shift factor at the origin, we fixed the temperature with the local Rindler temperature. Since $\mathrm{dS}_{d}$ is not asymptotically flat, the exact entropy-area relation should be reproduced by employing the Bousso-Hawking temperature [13] instead of the standard normalization of the present work. It is also worth trying to reproduce the relation with different thermodynamic definitions such as quasi-local energy, Wald entropy, and so on.

4. The total energy of the non-perfect fluid of our consideration agrees with the horizon energy defined in Ref. [6], so that the thermodynamic law holds in the same form. However, the large deficit solid angle leads to the discrepancy between two energy values and in the large deficit solid angle limit. It is beyond our consideration in this present work.

5. The pressure term in the first law is written only in terms of the vacuum expectation value of the scalar field. Since we did not vary the cosmological constant and fixed it in the theory, its pressure term did not appear as in the first derivation of the thermodynamic law for the cosmological horizon [5]. Even though we include the energy and pressure contributions from the cosmological constant, both effects are exactly canceled.

6. In the Schwarzschild geometry, the first law of black hole thermodynamics with a global monopole can also be checked in the same limit.

\section{Acknowledgments}

This research was supported by Basic Science Research Program through the National Research Foundation of Korea (NRF) funded by the Ministry of Science, ICT and Future Planning (Grant No. NRF2016R1D1A1B03931090).

\section{References}

[1] J. D. Bekenstein, Phys. Rev. D 7, 2333 (1973).

[2] J. M. Bardeen, B. Carter and S. W. Hawking, Commun. Math. Phys. 31, 161 (1973).

[3] S. W. Hawking, Commun. Math. Phys. 43, 199 (1975) Erratum: [Commun. Math. Phys. 46, 206 (1976)].

[4] S. Carlip, Int. J. Mod. Phys. D 23, 1430023 (2014) [arXiv:1410.1486 [gr-qc]].

[5] G. W. Gibbons and S. W. Hawking, Phys. Rev. D 15, 2738 (1977).

[6] T. Padmanabhan, Class. Quant. Grav. 19, 5387 (2002) [gr-qc/0204019].

[7] A. Corichi and A. Gomberoff, Phys. Rev. D 69, 064016 (2004) [hep-th/0311030].

[8] M. Barriola and A. Vilenkin, Phys. Rev. Lett. 63, 341 (1989).

[9] D. Harari and C. Lousto, Phys. Rev. D 42, 2626 (1990).

[10] Y. Kim, K. Maeda and N. Sakai, Nucl. Phys. B 481, 453 (1996) [gr-qc/9604030].

[11] S. Deser, R. Jackiw and G. 't Hooft, Annals Phys. 152, 220 (1984).

[12] M. Spradlin, A. Strominger and A. Volovich, hep-th/0110007.

[13] R. Bousso and S. W. Hawking, Phys. Rev. D 54, 6312 (1996) [gr-qc/9606052]. 